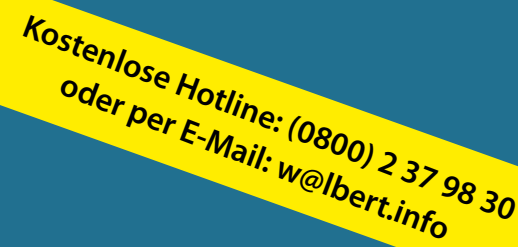

Leser der MMW können sich mit allen Fragen zur Abrechnung und Praxisführung an Helmut Walbert, Facharzt für Allgemeinmedizin, Würzburg, wenden. Sie erreichen ihn jeden Donnerstag von 13 bis 15 Uhr unter der kostenlosen Rufnummer (0800) 2379830 oder per E-Mail: w@lbert.info.

Helmut Walbert

Allgemeinarzt, Medizinjournalist und Betriebswirt Medizin

\title{
Gelten da keine Fachgebietsgrenzen?
}

Dr. med. M. N., Arzt für Allgemeinmedizin, München:

Ist es richtig, dass im organisierten Notfalldienst keine Fachgebietsgrenzen gelten?

Antwort: Dies ist richtig (siehe auch Beitrag auf S. 8). Diese Ausnahmeregelung gilt allerdings nur für den durch die Kassenärztliche Vereinigung (KV) organisierten Notfalldienst (NFD). Darunter fallen regional ganz unterschiedliche Zeiträume. In der Regel zählen die Wochenenden, Feiertage und der Mittwochnachmittag dazu. Es gibt aber auch KV-organisierte Notfalldienste während der gesamten Woche. Diese Fälle fallen dann auch unter die Ausnahmeregel.

In der Präambel zum Kapitel 1.2 „Versorgung im Notfall und im organisierten ärztlichen Not(-fall)dienst“ steht: „Die Nr. 1.5 der Allgemeinen Bestimmung gilt für die Berechnung von im Rahmen der Notfallversorgung erbrachten Gebührenordnungspositionen (GOP) nicht."

In 1.5 ist bekanntlich das abrechenbare Leistungsspektrum arztgruppenspezifisch beschränkt: „Arztgruppenspezifische GOP können nur von den in der Präambel des entsprechenden Kapitels bzw. Abschnitts genannten Vertragsärzten berechnet werden, sofern sie die dort aufgeführten Kriterien erfüllen oder einen Arzt angestellt haben, der die dort aufgeführten Kriterien erfüllt."

Für den Allgemeinarzt wird damit eine ganze Reihe von Leistungen im Notfalldienst berechnungsfähig, die sonst in die Versichertenpauschale fallen. Beispielsweise seien hier das notwendige Wechseln oder Legen eines Blasenkatheters GOP 02323 oder GOP 02100 (Infusion) genannt. Diese

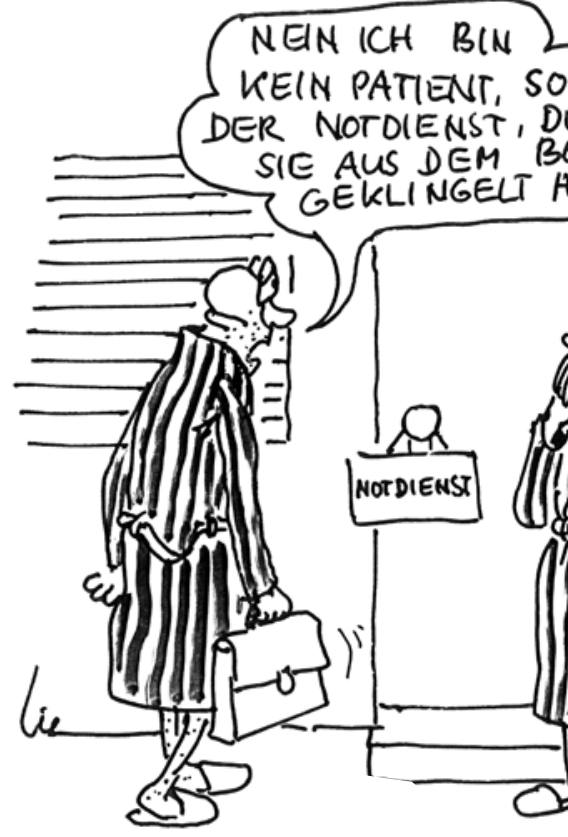

fallen im Alltag des Hausarztes in die Versichertenpauschale und sind daher nicht zusätzlich berechnungsfähig. Im organisierten NFD sind diese Leistungen nicht Inhalt der Notfallpauschale GOP 01210 und somit berechnungsfähig.

Wichtig ist es, dass das Bewusstsein erhalten bleibt, dass im NFD Leistungen aus allen Kapiteln des EBM abrechenbar sind, wenn diese zur Behandlung erforderlich sind: So aus dem Kapitel HNO: Tamponade bei starkem Nasenbluten GOP 09310 oder GOP 09361 „Kleinchirurgischer Eingriff II im Hals-Nasen-Ohren-Mund-Bereich und/oder primäre Wundversorgung im Hals-NasenOhren-Mund-Bereich bei Primärer Wundversorgung und/oder Entfernung festsitzender Fremdkörper aus dem Hals-Nasen-Ohren-

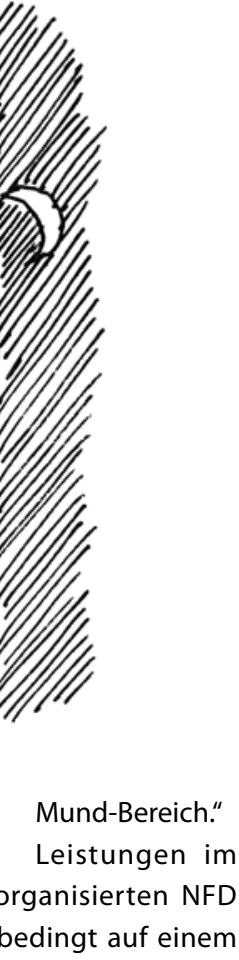

müssen unbedingt auf einem Notfall-Behandlungsschein nach Muster 19a abgerechnet werden. In der Regel werden die Leistungen im NFD ohne Mengenbegrenzung und außerhalb des fachgruppenspezifischen Topfes und Regelleistungsvolumens (RLV) vergütet. Nicht zum organisierten NFD zählt der organisierte kollegiale Vertretungsdienst während der Woche, wie er in vielen Regionen von Ärzten außerhalb des KV-organisierten NFD vereinbart ist.

Bezüglich der Wirtschaftlichkeit der Abrechnung und notwendiger Verordnung ist es wichtig zu wissen: Auch Fälle des organisierten NFD zählen mit zu den Behandlungsfällen und lösen somit alle fallzahlabhängigen Regelungen mit aus wie beispielsweise Arzneimittel- oder Heilmittel-Größen. 